CERN-TH.7518/94

CTP-TAMU-64/94

ACT-19/94

\title{
NUMBER OF GENERATIONS IN FREE FERMIONIC STRING MODELS
}

\author{
Ioannis Giannakis $^{(a),(b)}$, D.V. Nanopoulos ${ }^{(a),(b),(c)}$ and Kajia Yuan ${ }^{(a),(b)}$ \\ ${ }^{(a)}$ Center for Theoretical Physics, Texas A\&M University \\ College Station, TX 77843-4242, USA \\ (b) Astroparticle Physics Group, Houston Advanced Research Center (HARC) \\ The Mitchell Campus, The Woodlands, TX 77381, USA \\ (c) CERN Theory Division, 1211 Geneva 23, Switzerland
}

\begin{abstract}
In string theory there seems to be an intimate connection between spacetime and world-sheet physics. Following this line of thought we investigate the family problem in a particular class of string solutions, namely the free fermionic string models. We find that the number of generations $N_{g}$ is related to the index of the supersymmetry generator of the underlying $N=2$ internal superconformal field theory which is always present in any $N=1$ spacetime supersymmetric string vacuum. We also derive a formula for the index and thus for the number of generations which is sensitive to the boundary condition assignments of the internal fermions and to certain coefficients which determine the weight with which each spin-structure of the model contributes to the one-loop partition function. Finally we apply our formula to several realistic string models in order to derive $N_{g}$ and we verify our results by constructing explicitly the massless spectrum of these string models.
\end{abstract}


Number of Generations in Free Fermionic String Models ...

\section{Introduction.}

Recent experimental results indicate that the number of families with light left-handed neutrinos is three. Although this result suggests that additional families require neutrino components with $m_{\nu_{L}} \geq 45 \mathrm{GeV}$, the problem in understanding why nature has chosen to fit the observed fermions into three generations with identical quantum numbers remains. This is the so called family problem and it is the modern version of Rabi's question "Who ordered the muon?".

Superstring theory is the only viable candidate for a consistent, unified description of all interactions in nature. It is thus interesting to explore the possible solution of the family problem in this particular framework. Unfortunately although great progress has been made towards understanding the structure of string theory, it is fair to say that a full understanding is still lacking. What we seem to understand in string theory is how to construct solutions of the theory: any two-dimensional conformal field theory with the appropriate central charge represents a solution of the theory. Of special importance is a class of conformal field theories with $N=2$ world-sheet supersymmetry, since they give rise to $N=1$ spacetime supersymmetry. During the past several years a large number of solutions of string theory has been constructed. Some of these solutions can be viewed as compactifications of a ten-dimensional theory (Calabi-Yau manifolds, orbifolds..) and as such have a geometric interpretation while others are formulated directly in four dimensions (free fermionic models, lattice constructions, $N=2$ superconformal models) with no apparent geometric interpretation.

In its present formulation string theory cannot adress the problem of why nature has chosen to triplicate itself. At best we hope to gain an insight into the problem by attempting to relate the number of families of a particular string vacuum with some characteristic of the underlying superconformal structure on the world-sheet. In the Calabi-Yau [1] and orbifold [2] compactifications the number of families is related to a topological quantity, the Euler characteristic of the compactified internal space. Similar results towards this direction have been obtained for L-G models in [3]. The free fermionic string models constitute a class of vacua of string theory in which the extra degrees of freedom are realized in terms of free world-sheet fermions. Subsequently these vacua lack a geometric interpretation, although the information concerning the observable four-dimensional physics remains encoded in the internal superconformal field theory. As a result the family problem has remained largely unexplored in this particular class of solutions despite attempts to address it in [4].

It is the aim of this paper to address the family problem in the free fermionic string models. We will find that the number of families is given by the index of the supersymmetry generator of the underlying $N=2$ internal superconformal theory which is always present in any string vacuum with $N=1$ spacetime supersymmetry [5]. In the next section we discuss the supersymmetric index [6] of an $N=2$ superconformal theory [7]. This index can 
be expressed as a path integral over the degrees of freedom of the theory. In section 3 we will briefly review the free fermionic string models in order to establish the necessary notation. We will also derive an expression for the index of the internal $N=2$ superconformal field theory in terms of the contributions of the internal fermions of the model to the partition function, and provide arguments of why this formula gives us the number of families of a particular string vacuum. The expression for the index depends only on the boundary condition assignments of the internal fermions and a set of certain coefficients, which represent the weights with which each sector of the string model contributes to the one-loop string partition function. Subsequently in section 4 we will apply this formula to a class of realistic free fermionic string models with $N=1$ spacetime supersymmetry and derive the number of generations $N_{g}$ for these models. We will verify our results by calculating explicitly the massless spectrum of these models. Finally section 5 will summarize our results.

\section{The index of $N=2$ superconformal algebra.}

An $N=2$ superconformal algebra is an extension of the Virasoro algebra. In addition to the stress-energy tensor $T(z)$, its generators include two spin- $\frac{3}{2}$ fields (the supercurrents) $T_{\mathrm{F}}^{ \pm}(z)$, and a spin-1 field $J(z)$, the generator of a $U(1)$ Kac-Moody algebra. These fields can be expanded in terms of Laurent modes as follows: $T(z)=\sum z^{-2-n} L_{n}, T_{\mathrm{F}}^{ \pm}(z)=$ $\sum z^{-\frac{3}{2}-n \mp a} G_{n \pm a}^{ \pm}$and $J(z)=\sum z^{-1-n} J_{n}$, where $n \in \mathbf{Z}$, and the parameter $a \in[0,1)$ labels the boundary conditions of the fermionic operators $T_{\mathrm{F}}^{ \pm}$. In particular for the Ramond (R) sector $a=0$, and for the Neveu-Schwarz (NS) sector $a=\frac{1}{2}$. The $N=2$ superconformal algebra can be expressed either in terms of the operator product expansions of these fields or equivalently in terms of the (anti)-commutation relations of their modes. In terms of the modes the algebra is given

$$
\begin{aligned}
& {\left[L_{m}, L_{n}\right]=(m-n) L_{m+n}+\frac{c}{12}\left(m^{3}-m\right) \delta_{m+n}, \quad\left[J_{m}, J_{n}\right]=\frac{c}{3} m \delta_{m+n},} \\
& \left\{G_{m+a}^{+}, G_{n-a}^{-}\right\}=L_{m+n}+\frac{1}{2}(m-n+2 a) J_{m+n}+\frac{c}{6}\left[(m+a)^{2}-\frac{1}{4}\right] \delta_{m+n}, \\
& {\left[L_{m}, G_{n \pm a}^{ \pm}\right]=\left(\frac{m}{2}-n \mp a\right) G_{m+n \pm a}^{ \pm}, \quad\left[L_{m}, J_{n}\right]=-n J_{m+n}, \quad\left[J_{m}, G_{n \pm a}^{ \pm}\right]= \pm G_{m+n \pm a}^{ \pm}}
\end{aligned}
$$

Here $c$ is the central charge of the algebra. For any given $a \in[0,1)$, the complete representation space of the corresponding $N=2$ superconformal algebra can be obtained by acting successively with $L_{-n}, J_{-n}, G_{-r}(n, r>0)$ on the highest weight states $|\phi\rangle$, states which are annihilated by $L_{n}, G_{r}, J_{n}$ for any $n, r>0$ and are eigenstates of the Cartan generators $L_{0}$ and $J_{0}$ with eigenvalues $h$ and $q$ respectively: $L_{0}|\phi\rangle=h|\phi\rangle, J_{0}|\phi\rangle=q|\phi\rangle$.

The fact that an $N=2$ superconformal algebra outlined above entails the notion of an index can be most easily seen by considering the subalgebra generated by $L_{0}, J_{0}$ and $G_{0}^{ \pm}$ $\left(G_{0}^{-}=G_{0}^{+\dagger}\right)$ in the Ramond sector [7], where $h \geq \frac{c}{24}$ due to unitarity constraints. There 
are two types of states in the Hilbert space: (a) massive states $\left(h>\frac{c}{24}\right)$ which come in pairs (i.e. $|h, q\rangle$ and $G_{0}^{+}|h, q\rangle$ ) with the same mass, since $\left[L_{0}, G_{0}^{ \pm}\right]=0$ and opposite eigenvalues of $(-1)^{J_{0}}$ since $\left\{(-1)^{J_{0}}, G_{0}^{ \pm}\right\}=0$; and (b) massless states $\left(h=\frac{c}{24}\right)$ which are isolated. The latter are annihilated by $G_{0}^{ \pm}$: they are the supersymmetric ground states of the theory. This situation is akin to the one which led Witten to introduce the notion of the supersymmetric index in order to probe issues of supersymmetry breaking[6]. Then $G_{0}^{+}$corresponds to the "supersymmetry charge" $Q, L_{0}$ corresponds to the "Hamiltonian" $Q^{2}$, and finally the operator $(-1)^{J_{0}}$ plays the role of the "chirality operator" $(-1)^{F}$. Thus, for an $N=2$ superconformal algebra, one can define [7] the index of $G_{0}^{+}$as ind $\left(G_{0}^{+}\right)=\operatorname{Tr}(-1)^{J_{0}}$. $^{1}$

There is one caveat in the above definition which is related with the eigenvalues of $J_{0}$. Strictly speaking, it is only when $(-1)^{J_{0}}= \pm 1$ that one can meaningfully define $(-1)^{J_{0}}$ as a certain "chirality operator". Under such condition, the representation space of the Ramond sector decomposes into two subspaces $V_{+} \oplus V_{-}$characterized by the action of $(-1)^{J_{0}}$, with $(-1)^{J_{0}} V_{ \pm}= \pm V_{ \pm}$. As a consequence the massless states split into those in $\operatorname{ker}\left(G_{0}^{+}\right) \subset V_{+}$, which are annihilated by $G_{0}^{+}$and those in $\operatorname{ker}\left(G_{0}^{-}\right) \subset V_{-}$, which are annihilated by $G_{0}^{-}$, and $\operatorname{Tr}(-1)^{J_{0}}$ coincides with $\operatorname{dim} \operatorname{ker}\left(G_{0}^{+}\right)-\operatorname{dim} \operatorname{ker}\left(G_{0}^{-}\right)$. The generic structure of the $N=2$ superconformal algebra alone does not guarantee the eigenvalues of $J_{0}$ to be integers, so in general $\operatorname{Tr}(-1)^{J_{0}}$ may be ill-defined as an index. Fortunately, this caveat does not seem to present a real obstacle for us. For heterotic string vacua, the presence of $N=1$ space time supersymmetry requires that the local $(1,0)^{2}$ superconformal symmetry should be promoted to a global $(2,0)$. If one considers the total left-moving $N=2$ superconformal algebra, which contains both the space-time and internal degrees of freedom $(c=15)$, then the $U(1)$ charges (eigenvalues of $J_{0}$ ) of all physical states are guaranteed to be integers. For reasons that will become clear below, one often is interested in the $(2,0)$ superconformal theories $(c=9)$ that describe only the internal degrees of freedom of the classical vacua of the heterotic string. In that case, the eigenvalues of $J_{0}$ are half integers in the Ramond sector. Neverthless, it is possible to re-define the corresponding index ind $\left(G_{0}^{+}\right)$in terms of the trace of an operator $(-1)^{J_{0}^{\prime}}$ which preserves the algebraic properties of $(-1)^{J_{0}}$ and has integer eigenvalues. We will discuss an explicit example in the next section when we consider the free fermionic string models. In the rest of this section, we will write formally the index operator as the $\operatorname{Tr}(-1)^{J_{0}}$. For applications in string theory, vacua with left-moving $(2,0)$ supersymmetry, the trace needs to be properly regularized and the appropriate object to consider is [7]

$$
\operatorname{ind}\left(G_{0}^{+}\right)=\operatorname{Tr}\left[(-1)^{J_{0}} q^{\left(G_{0}^{+}+G_{0}^{-}\right)^{2}} \bar{q}^{\bar{L}_{0}-\frac{c}{24}}\right]=\operatorname{Tr}\left[(-1)^{J_{0}} q^{L_{0}-\frac{c}{24}} \bar{q}^{\bar{L}_{0}-\frac{c}{24}}\right]
$$

where the trace runs over only the Ramond sector, $q=e^{2 \pi i \tau}$ and $\bar{L}_{0}$ is the right-moving Hamiltonian.

1 For any value $a \neq 0$, the index of the corresponding $N=2$ superconformal algebra can be defined via "spectral flow" [8].

2 In the convention we use, the left-moving sector is supersymmetric, whereas the rightmoving sector is not. 
The above discussion indicates that, for any heterotic string model with $N=1$ spacetime supersymmetry, one can always define an index for the supersymmetry generator of the underlying left-moving $N=2$ superconformal algebra (2.2). We would like to emphasize that this is true for the full class of heterotic string vacua with $N=1$ space-time supersymmetry, which includes both the familiar $(2,2)$ models and the more generic $(2,0)$ ones. Furthermore, in the current approach, the question of whether or not the internal sectors of these string models admit some geometric interpretation becomes secondary.

We now turn to the family problem in these string models. Let us first recall that, although one speaks about light chiral fermions, it is the space-time chirality associated with the four-dimensional Dirac operator that is really referred to, in string theory, the consistency of the theory does establish a correlation between the space-time chirality and the internal chirality. In fact, the number of light chiral fermion generations is given by the index of the internal Dirac operator ind $(\not D)^{\text {int }}=\operatorname{Tr} \Gamma^{\text {int }}$. If the internal sector of the string model can be viewed as a compact six-dimensional manifold $K$, (Calabi-Yau compactifications with standard embedding and $(2,2)$ symmetric orbifold compactifications), one can further establish a relation between the index of the internal Dirac operator and the Euler characteristic $\chi(K)$ of $K$. For more general string vacua whose internal sectors lack any geometric interpretation, the concept of the Euler characteristic becomes not directly applicable. However, going back to the root of the problem, we see that the crucial element here is the index of the internal Dirac operator, rather than the Euler characteristic. But how can one have a handle on the former in general? Amazingly, the answer to this question is provided by the index of the $N=2$ superconformal algebra discussed above. In fact, it was shown in Ref. [7] that, in the presence of $N=1$ space-time supersymmetry, the index $\operatorname{Tr}(-1)^{J_{0}}$ is identical to the index of the Dirac operator $\operatorname{Tr} \Gamma$. More specifically the index $\operatorname{Tr}(-1)^{J_{0}}$ provides the number of massless states of a given eigenvalue of $(-1)^{J_{0}}$ minus the number of massless states of the opposite eigenvalue. In a particular string vacuum $(-1)^{J_{0}}$ measures the internal chirality of a particle state. Consistency of the theory imposes a correlation between $(-1)^{J_{0}}$ and the chirality of a particle state as it is measured by an experimentalist in our world. As a result the only contribution to the index arises from chiral massless states (leptons, quarks..) while massless vector-like particles (Higgs, gravitinos, gauginos..) do not contribute. Since for string models with $N=1$ space-time supersymmetry their internal sectors realize an $N=2$ superconformal algebra $((2,0)$ world-sheet supersymmetry) with $c=9$, the natural object to consider therefore is the index (2.2) for the internal sectors.

\section{Index formula in free fermionic string models.}

In the previous section, we have associated the number of families in any $N=1$ spacetime supersymmetric four-dimensional heterotic string model with the index (2.2) of the underlying internal left-moving $N=2$ superconformal algebra. We now focus on a particular class of such four-dimensional heterotic string models, namely the free fermionic string models [9][10][11]. In the free fermionic constructions the internal degrees 
of freedom are realized in terms of free world-sheet fermions. In the light-cone gauge, the two transverse space-time coordinates $X^{\mu}$ and their left-moving superpartners $\psi^{\mu}$ are supplemented by real or complex left and right-moving free world-sheet fermions. These fermionic degrees of freedom furnish a representation of an internal left-moving $N=1$ superconformal theory with $c_{L}=9$ and a right-moving conformal theory with $c_{R}=22$. For instance, in a particularly interesting class of models, the left-moving internal sector can be specified in terms of the 18 real fermions being arranged into six sets $\left(\chi^{I}, y^{I}, \omega^{I}\right)$ $(I=1, \cdots, 6)$, each transforming in the adjoint representation of $S U(2)$, such that the local world-sheet supersymmetry is non-linearly realized among them. The supercurrent is then given by the following expression [12]:

$$
T_{\mathrm{F}}(z)=\psi^{\mu} \partial X_{\mu}+i \sum_{I} \chi^{I} y^{I} \omega^{I}
$$

As for the right-moving internal sector, in general one can use $n_{r}$ real fermions and $n_{c}$ complex fermions, as long as $n_{r}+2 n_{c}=44$.

The essential ingredient in the free fermionic formulation is the spin-structures of all the fermions, namely the possible boundary condition assignments for the fermions as they are parallel transported around the two non-contractible loops ( $\sigma, \tau$ directions) of a torus. It is convenient to use a vector, which we refer to as a spin-structure vector, to represent a set of boundary conditions for all the world-sheet fermions. We follow the notation of the second paper in Ref. [10]. For instance, an entry 1(0) in spin-structure vectors means that the corresponding fermion has periodic (anti-periodic) boundary condition. In this construction, a particular string model is completely specified by: (a) a basis $\mathcal{B}=$ $\left\{b_{1}, b_{2}, \ldots b_{n}\right\}$ of some vectors which generates all consistent spin-structures of the model. These vectors altogether form a finite additive group $\Xi$ of dimension $M=\prod_{i=1}^{n} N_{b_{i}}$, where $N_{b_{i}}$ is the order of the basis vector $b_{i}$ and (b) a set of spin-structure coefficients $C\left[\begin{array}{l}b_{i} \\ b_{j}\end{array}\right]$ for a pair of basis vectors $b_{i}, b_{j} \in \mathcal{B}$, which determines the relative weights with which all possible spin-structures contribute to the one-loop string partition function. Consistency conditions of string theory, such as modular invariance etc., can be cast into a set of simple rules for the basis vectors $b_{i} \in \mathcal{B}$ and the coefficients $C\left[\begin{array}{l}b_{i} \\ b_{j}\end{array}\right][9][10]$.

The total Hilbert space of the string model consists of $M$ sectors, each corresponding to an element of $\Xi$, i.e., for every $\alpha \in \Xi$ there is a $\alpha$-sector. The set of all sectors can be divided into two classes $\Xi^{+}$and $\Xi^{-}$, each containing $M / 2$ sectors, depending on the value of $\delta_{\alpha}=e^{i \pi \alpha\left(\psi^{\mu}\right)}= \pm 1$. Since the bosonic or fermionic nature of the particle state is solely determined by the boundary conditions of the world-sheet fermions with spacetime index $\psi^{\mu}$, if $\alpha \in \Xi^{+}$, the $\alpha$-sector provides space-time bosons, while if $\alpha \in \Xi^{-}$, it provides space-time fermions. In a given $\alpha$-sector, the spin-structures have the same assignment in the $\sigma$-direction (around the string) determined by the vector $\alpha$, while their assignments along the $\tau$-direction can still be allowed to vary according to any vector $\beta \in \Xi$. For every $\alpha$-sector, the summation over contributions to one-loop string partition function from all such $\beta$ in $\tau$-direction realizes a GSO-type projection, which is equivalent to a set 
of separate GSO-projections, one for each basis vector. Finally, the GSO-projections for a specific $\alpha$-sector determines the physical states in that sector.

In order to consturct a fermionic string model with $N=1$ space-time supersymmetry, it is important to have sectors that could give rise to massless gravitinos. This can be achieved by including one such vector ( $S$-vectors) [13] in our basis. The simplest choice is given by

$$
S=\left(1100100100100100100: \mathbf{0}_{R}\right) .
$$

In our notation, the first entry is reserved for the two transverse $\psi^{\mu}$ treated as a single complex fermion, the following 18 entries correspond to the left-moving internal fermions $\left(\chi^{I}, y^{I}, \omega^{I}\right)$. This is the only $S$-vector which permits the realization of the left-moving internal sector entirely in terms of real fermions, all other $S$-vectors given in Ref. [13] require the use of complex fermions. This particular choice for the $S$-vector only guarantees that the model will have $N=4$ space-time supersymmetry. Subsequently one needs to introduce other basis vectors in order to reduce space-time supersymmetry from $N=4$ to $N=1$. We would like to emphasize that the choice of coefficients $C\left[\begin{array}{c}S \\ b_{i}\end{array}\right]$ for any $b_{i} \in \mathcal{B}$ is also very crucial, because it is possible that even if the model has the correct basis vectors in order to generate $N=1$ space-time supersymmetry, some otherwise consistent choice of certain $C\left[\begin{array}{c}S \\ b_{i}\end{array}\right]$ could result in the elimination of the gravitinos from the spectrum through the GSO-projections. There is only a limited number of choices for the $C\left[\begin{array}{l}S \\ b_{i}\end{array}\right]$ that one can make. In fact, given the above $S$-vector, the sufficient condition for $N=1$ supersymmetry is $[14]$

$$
C\left[\begin{array}{l}
S \\
b_{i}
\end{array}\right]=-\delta_{b_{i}} .
$$

This condition in the free fermionic formulation is analogous to the condition for the vanishing of the first Chern class in Calabi-Yau or orbifold compactifications.

For free fermionic models with $N=1$ space-time supersymmetry, the left-moving local $(1,0)$ superconformal symmetry can be extended to a global $(2,0)$ one. In general, the precise realization of this $(2,0)$ structure in the fermionic models is contigent on some details of the models, the key issue being how to construct the $U(1)$ current $J(z)$ in terms of the world-sheet fermions. With the $S$-vector given as (3.2), the $U(1)$ current $J(z)$ of the internal left-moving $N=2$ superconformal algebra can be constructed in terms of the six periodic fermions $\chi^{I}$ in the $S$-vector, as follows [15]

$$
J(z)=i \partial_{z}\left(S_{12}+S_{34}+S_{56}\right)
$$

where $S_{12}$ is the bosonization of the complex fermion $\chi^{12}$ defined as $e^{i S_{12}}=\chi^{12}=\frac{1}{\sqrt{2}}\left(\chi^{1}+\right.$ $i \chi^{2}$ ), and similarly for $S_{34}$ and $S_{56}$. The internal part of the supercurrent $T_{\mathrm{F}}$ (see Eq. (3.1)) indeed splits into two pieces $T_{\mathrm{F}}^{ \pm}$with eigenvalues \pm 1 under the action of $J(z)$. Since in 
the Ramond sector of this internal $N=2$ superconformal algebra $T_{\mathrm{F}}^{ \pm}$are periodic, ${ }^{3}$ from Eq. (3.1) we see that in this sector $\psi^{\mu}$ should also be periodic. Therefore, in studying the index (2.2) of the internal $N=2$ superconformal algebra for this class of fermionic models, we only need to include the $\alpha$-sectors with $\delta_{\alpha}=-1$, namely, those $\alpha \in \Xi^{-}$.

However, for these sectors the eigenvalues of $J_{0}$ are half-integers instead of integers [15]. To see why this is the case, we first note that the eigenvalues of $J_{0}$ are the sum of the so-called fermionic charges [9] of the three complex fermions $\chi^{12}, \chi^{34}$ and $\chi^{56}$. We recall that the fermionic charge $Q_{\alpha}(f)$ of a complex fermion $f$ in a particular $\alpha$-sector is given by $[9][16]$

$$
Q_{\alpha}(f)=F_{\alpha}(f)+\frac{1}{2} \alpha(f)
$$

where $F_{\alpha}(f)$ is the fermion number and the second term represents the vacuum charge arising from filling the negative energy states. Thus, it is the vacuum charges involved in the definition of $J(z)$ which cause the problem here. Clearly then we can regulate $J_{0}$ by removing the vacuum charges, the proper operator $J_{0}^{\prime}$ that we alluded to in the previous section is then simply the sum of the fermion numbers of $\chi^{12}, \chi^{34}$ and $\chi^{56}$.

We now proceed with the computation of the index $\operatorname{Tr}(-1) J_{0}^{\prime}$ for free fermionic string models. To this end let us first recall that the index $\operatorname{Tr}(-1)^{F}$ of a general $1+1$ dimensional supersymmetric non-linear $\sigma$-model [6] can be computed as a path integral [17]

$$
\operatorname{Tr}(-1)^{F}=\int d X^{I}(\sigma, \tau) d \psi^{I}(\sigma, \tau) e^{-A}
$$

where $X^{I}(\sigma, \tau)$ represent the bosonic degrees of freedom and $\psi^{I}(\sigma, \tau)$ their superpartners. The integration is over periodic fermionic degrees of freedom in both $\sigma$ and $\tau$ directions due to the insertion of $(-1)^{F}$, and $A$ is the action of the supersymmetric non-linear $\sigma$-model. We note that in (3.6), $X^{I}$ and $\psi^{I}$ include both left-moving and right-moving degrees of freedom. Whenever it is possible to interpret the bosonic degrees of freedom $X^{I}$ of the non-linear sigma model as coordinates of a compact six-dimensional manifold $K$, as it is the case with Calabi-Yau and orbifold compactifications with standard embedding, equation (3.6) gives the Euler characteristic of $K$. However, ever since the advent of heterotic string theory, it became evident that string theory per se does not demand a symmetrical treatment between the left and right degrees of freedom. This is true for the asymmetric orbifolds [18] as well as for the free fermionic string models. Therefore we are led to a generalization of (3.6) given by

$$
\operatorname{Tr}(-1)^{J_{0}^{\prime}}=\int d X_{L}^{I} d X_{R}^{I} d \psi_{L}^{I} d \psi_{R}^{I} e^{-A} .
$$

3 We remind the cautious readers that the usual analysis of fermionic formulation is carried out on the cylinder, whereas the presentation of the previous section is given on the complex plane. 
We would like to emphasize that (3.7) indeed generalizes (3.6). The point is that, since in (3.7) the operator $J_{0}^{\prime}$ is defined interms of $\psi_{L}^{I}$, only the left-moving world-sheet supersymmetry is needed. In other words, it is not necessary to have right-moving supersymmetry among $X_{R}^{I}$ and $\psi_{R}^{I}$ for (3.7) to make sense. In short, (3.7) is valid for any $(2,0)$ models, while (3.6) is valid only for $(2,2)$ models.

For free fermionic models, the fields $X_{L}^{I}$ and $\psi_{L}^{I}$ in (3.7) obviously correspond to the 18 left-moving fermions $\left(\chi^{I}, y^{I}, \omega^{I}\right)$. In fact, given the form of the $S$-vector, it is easy to see that each pair $\left(y^{I}, \omega^{I}\right)$ can be viewed as the fermionization of a bosonic field $X_{L}^{I}$, and $\chi^{I}$ are simply the corresponding $\psi_{L}^{I}$. What appears to be uncertain is the representation of the right-moving degrees of freedom $X_{R}^{I}$ and $\psi_{R}^{I}$ in terms of some right-moving worldsheet fermions. If $m_{r}$ real fermions and $m_{c}$ complex fermions are included, one must have $m_{r}+2 m_{c}=18$ to match the right-moving degrees of freedom. Moreover, for a fermionic model with a $(2,2)$ superconformal structure, the right-moving fermions in (3.7) obviously should be those which realize the global right-moving $N=2$ superconformal algebra, because in that case (3.7) is identical to (3.6). This observation enables one to determine these internal right-moving fermions in some $(2,0)$ models as well. Suppose one is given a fermionic model with a basis $\mathcal{B}$, which has $N=1$ space-time supersymmetry but only $(2,0)$ world-sheet supersymmetry. One then can investigate all the normal subsets of $\mathcal{B}$ that would also give rise to models with $N=1$ space-time supersymmetry. If one can find a sub-model which actually admits a $(2,2)$ structure, then the right-moving fermions that are responsible for the presence of the additional $(0,2)$ structure in this sub-model are the ones that should be included in $(3.7)$ for the index of the original $(2,0)$ model. This procedure may sound fairly long-winded at this point but, in reality, it is not. We will provide some examples in the next section in order to demonstrate how this is done in practice. Of course, we are aware of the fact that maybe not all of the $(2,0)$ fermionic models are bound to have a $(2,2)$ "core" in the sense we have just described. In that case, the problem of deciding which right-moving fermions should be included in (3.7) remains.

Having specified all the fields in (3.7), we can proceed to compute the path integral. The calclulation is very similar to that of the one-loop fermionic partition function [9][10]. As in the case of orbifolds, in the free fermionic models one is bounded to integrate over field configurations with twisted boundary conditions, namely over different spin-structures for the appropriate fermions. In other words, the index $\operatorname{Tr}(-1)^{J_{0}^{\prime}}$ is in this case a charactervalued index $\operatorname{Tr} g(-1)^{J_{0}^{\prime}}$, where $g$ twists the boundary conditions of the internal worldsheet fermions in both $\sigma$ and $\tau$ directions. Since only massless states contribute to the index, the total contribution from different $\sigma$-twists manifests itself as a sum over all massless $\alpha$-sectors with $\alpha \in \Xi^{-}$. For each of such $\alpha$-sector, similar to the orbifold case, the contributions from the $\tau$-twists form a projection onto invariant states. The insertion of $(-1)^{J_{0}^{\prime}}$ further implies that the only $\tau$-twists which give non-zero contribution are those given by the vectors $\beta \in \Xi^{-}$. There are $M / 2$ such $\beta$ vectors, so the projection amounts to a summation over them divided by $M / 2$. In addition, we expect the index $\operatorname{Tr}(-1) J_{0}^{\prime}$ being a topological quantity, to remain invariant under modular transformations. Putting all these 
pieces together, we derive a formula for the index of the underlying $N=2$ superconformal theory in the free fermionic string models given by

$$
\operatorname{Tr}(-1)^{J_{0}^{\prime}}=\frac{2}{\prod_{i} N_{b_{i}}} \sum_{\alpha \in \Xi^{-}}^{\prime} \sum_{\beta \in \Xi^{-}} C\left[\begin{array}{l}
\alpha \\
\beta
\end{array}\right] Z^{\text {int }}\left[\begin{array}{l}
\alpha \\
\beta
\end{array}\right] .
$$

Here the prime on $\alpha$-summation sign indicates that only massless Ramond sectors contribute. The spin-structure coefficients $C\left[\begin{array}{l}\alpha \\ \beta\end{array}\right]$ in (3.8) are the same as those appearing in the one-loop string partition function, which can be completely determined by the basic

set of $C\left[\begin{array}{l}b_{i} \\ b_{j}\end{array}\right]$. Finally, $Z^{\text {int }}\left[\begin{array}{l}\alpha \\ \beta\end{array}\right]$ represents the product of all determinants of the world-sheet Dirac operators for the internal fermions, with $\sigma$ - and $\tau$-boundary conditions specified by $\alpha$ and $\beta$, respectively. Using our phase convention, the determinant of a single complex left-moving fermion $f$ with $\alpha(f)=a$ and $\beta(f)=b$ is given in terms of the $\Theta$ function as

$$
Z\left(\begin{array}{l}
a \\
b
\end{array}\right)=\frac{e^{i \pi(a b) / 2}}{\eta(\tau)} \Theta\left[\begin{array}{c}
a / 2 \\
-b / 2
\end{array}\right](0 \mid \tau),
$$

where $\eta(\tau)$ is the Dedekind function. The determinant of a single complex right-moving fermion is obtained by taking the complex conjugate of (3.9). For real fermions, one needs to take the square root of these expressions. The right hand side of the index formula (3.8) can be either positive or negative and as we have argued in the previous section it provides a measure of the space-time chiral asymmetry in the free fermionic string models. Its absolute value then provides the number of generations $N_{\text {gen }}$ in such models.

\section{Some illustrative examples.}

The purpose of this section is two-fold. First, we would like to apply the index formula (3.8) presented in the previous section in particular string models and demonstrate explicitly how to choose the internal right-moving fermions and second we would like to verify the results obtained in this manner by comparing them with the results which arise by direct derivation of the massless spectrum of these models.

Let us first start with a simple basis $\mathcal{B}_{1}=\left\{\mathbf{1}, S, b_{1}, b_{2}, b_{3}\right\}$, where in vector $\mathbf{1}$ all world-sheet fermions are periodic, vector $S$ is given by (3.2) and

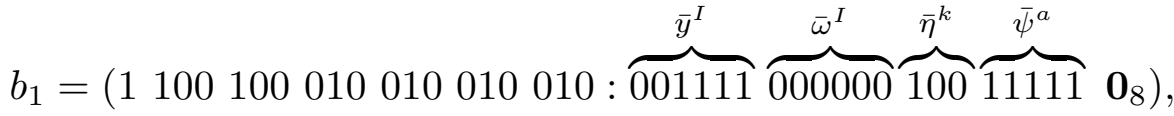

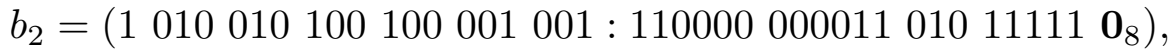

$$
\begin{aligned}
& b_{3}=\left(\begin{array}{ll}
1 & 001001001001100100: 00000011110000111111 \mathbf{0}_{8}
\end{array}\right) \text {. }
\end{aligned}
$$

This set of basis vectors was first introduced in the construction of the flipped $S U(5)$ model in Ref. [16] and have been adopted in many other free fermionic models. In writing these 
spin-structure vectors we have separated the right-moving fermions into 12 real fermions $\bar{y}^{I}, \bar{\omega}^{I}(I=1, \cdots, 6)$ while the rest are treated as complex fermions. In these vectors, the boundary conditions for each pair $\left(\bar{y}^{I}, \bar{\omega}^{I}\right)$ are identical to those for the corresponding pair $\left(y^{I}, \omega^{I}\right)$. Similarly, right-moving complex fermions $\bar{\eta}^{1,2,3}$ have the same boundary conditions as left-moving fermions $\chi^{12,34,56}$ in all vectors except for $S$. This simple observation prompts us to include these right-moving fermions $\left(m_{r}=12, m_{c}=3\right)$ in the index formula (3.8). In fact, it is easy to see that these fermions indeed realize an internal right-moving $N=2$ superconformal algebra, i.e., an additional global $(0,2)$ world-sheet supersymmetry. Upon bosonization the 12 real fermions $\left(\bar{y}^{I}, \bar{\omega}^{I}\right)$ provide the right-moving parts $X_{R}^{I}$ of the coordinates of the internal six-dimensional manifold $K$ while the $\bar{\eta}^{1,2,3}$ correspond to the degrees of freedom that realize the embedding of the spin connection into the gauge group.

In any fermionic model generated by the basis $\mathcal{B}_{1}$ there are $M / 2=2^{5} / 2=16$ vectors in $\Xi^{-}$, since $N_{b_{i}}=2$ for any $b_{i} \in \mathcal{B}_{1}$. These provide the following 16 sectors:

$$
\begin{aligned}
& \mathbf{1}(+\zeta), \quad S(+\zeta), \\
& b_{1}(+\zeta), \quad b_{2}(+\zeta), \quad b_{3}(+\zeta), \\
& S+b_{1}+b_{2}(+\zeta), \quad S+b_{2}+b_{3}(+\zeta), \quad S+b_{3}+b_{1}(+\zeta)
\end{aligned}
$$

Here for convenience we have introduced $\zeta \equiv \mathbf{1}+b_{1}+b_{2}+b_{3}$. The boundary conditions of the internal fermions in a particular vector are not affected by adding $\zeta$, thus

$$
Z^{\text {int }}\left[\begin{array}{l}
\alpha \\
\beta
\end{array}\right]=Z^{\text {int }}\left[\begin{array}{c}
\alpha+\zeta \\
\beta
\end{array}\right]=Z^{\text {int }}\left[\begin{array}{c}
\alpha \\
\beta+\zeta
\end{array}\right]=Z^{\text {int }}\left[\begin{array}{c}
\alpha+\zeta \\
\beta+\zeta
\end{array}\right] .
$$

Among the 16 sectors in (4.2) which give rise to space-time fermions, only $\left(S, S+\zeta, b_{1}, b_{2}, b_{3}\right)$ will produce massless fermions, so there are five terms in the $\alpha$-summation of the index formula (3.8). First, for any vector $\beta$ taken from the set (4.2), we have

$$
Z^{\text {int }}\left[\begin{array}{l}
S \\
\beta
\end{array}\right]=0
$$

This is because $Z^{\operatorname{int}}\left[\begin{array}{l}S \\ \beta\end{array}\right]$ always includes at least one factor $Z\left(\begin{array}{l}1 \\ 1\end{array}\right)$ (one left-moving real fermion with periodic boundary conditions in both $\sigma$ and $\tau$ directions) which vanishes according to Eq.(3.9)

$$
Z\left(\begin{array}{l}
1 \\
1
\end{array}\right)=e^{i \pi / 4} \sqrt{\frac{\theta_{1}(\tau)}{\eta(\tau)}}=0
$$

Therefore, the $S$-sector does not contribute to the index. The same is also true for the $(S+\zeta)$-sector because of (4.3). In fact, we know that these two sectors provide spacetime gravitinos and gauginos which are not chiral. In bosonic language these two sectors correspond to the untwisted sectors of the orbifold compactifications. This is in agreement with the fact that chiral fermions in the orbifold models arise from the twisted sectors. We would like to point out that, except for the condition (3.3) which guarantees the validity 
of the $N=2$ index itself, this particular result for the sectors $S$ and $(S+\zeta)$ is independent of the choice of the coefficients $C\left[\begin{array}{l}b_{i} \\ b_{j}\end{array}\right]$, therefore, it remains true for all models generated by the basis $\mathcal{B}_{1}$ with $N=1$ space-time supersymmetry.

We next calculate the contributions from the sectors $\left(b_{1}, b_{2}, b_{3}\right)$. Several terms in the $\beta$-summation vanish due to Eq. (4.5). For instance, making use of (4.3), the contribution of the $b_{1}$-sector is given by

$$
I_{b_{1}}=\frac{1}{16}\left(C\left[\begin{array}{l}
b_{1} \\
b_{2}
\end{array}\right]+C\left[\begin{array}{c}
b_{1} \\
b_{2}+\zeta
\end{array}\right]\right) Z^{\text {int }}\left[\begin{array}{l}
b_{1} \\
b_{2}
\end{array}\right]+\frac{1}{16}\left(C\left[\begin{array}{l}
b_{1} \\
b_{3}
\end{array}\right]+C\left[\begin{array}{c}
b_{1} \\
b_{3}+\zeta
\end{array}\right]\right) Z^{\text {int }}\left[\begin{array}{l}
b_{1} \\
b_{3}
\end{array}\right] \text {. }
$$

To compute this expression, we write the Dirac determinants in terms of the $\theta$-functions according to the following relations (see Eq. (3.9))

$$
Z\left(\begin{array}{l}
1 \\
0
\end{array}\right)=\sqrt{\frac{\theta_{2}(\tau)}{\eta(\tau)}}, \quad Z\left(\begin{array}{l}
0 \\
0
\end{array}\right)=\sqrt{\frac{\theta_{3}(\tau)}{\eta(\tau)}}, \quad Z\left(\begin{array}{l}
0 \\
1
\end{array}\right)=\sqrt{\frac{\theta_{4}(\tau)}{\eta(\tau)}}
$$

and then use the well-known Jacobi triplet product identity

$$
\theta_{2}(\tau) \theta_{3}(\tau) \theta_{4}(\tau)=2 \eta^{3}(\tau)
$$

The result is then (note $\bar{\eta}^{1,2,3}$ are complex)

$$
Z^{\text {int }}\left[\begin{array}{l}
b_{1} \\
b_{2}
\end{array}\right]=Z^{\text {int }}\left[\begin{array}{l}
b_{1} \\
b_{3}
\end{array}\right]=2^{6}
$$

As for the spin-structure coefficients, using the rules given in Ref. [10], we immediately get

$$
C\left[\begin{array}{c}
b_{1} \\
b_{2}+\zeta
\end{array}\right]=C\left[\begin{array}{l}
b_{1} \\
b_{3}
\end{array}\right], \quad C\left[\begin{array}{c}
b_{1} \\
b_{3}+\zeta
\end{array}\right]=C\left[\begin{array}{l}
b_{1} \\
b_{2}
\end{array}\right]
$$

Therefore, Eq. (4.6) reduces to

$$
I_{b_{1}}=8\left(C\left[\begin{array}{l}
b_{1} \\
b_{2}
\end{array}\right]+C\left[\begin{array}{l}
b_{1} \\
b_{3}
\end{array}\right]\right)
$$

Similarly, we have

$$
I_{b_{2}}=8\left(C\left[\begin{array}{l}
b_{2} \\
b_{3}
\end{array}\right]+C\left[\begin{array}{l}
b_{2} \\
b_{1}
\end{array}\right]\right), \quad I_{b_{3}}=8\left(C\left[\begin{array}{l}
b_{3} \\
b_{1}
\end{array}\right]+C\left[\begin{array}{l}
b_{3} \\
b_{2}
\end{array}\right]\right)
$$

for the $b_{2}$ - and $b_{3}$-sector, respectively.

So far, we have not yet specified the basic set of the coefficients $C\left[\begin{array}{l}b_{i} \\ b_{j}\end{array}\right]$, except for $C\left[\begin{array}{c}S \\ b_{i}\end{array}\right]$ which were chosen to satisfy (3.3). After we fix $C\left[\begin{array}{l}\mathbf{1} \\ \mathbf{1}\end{array}\right]=-1$, there are still six coefficients 
that can be independently chosen to be \pm 1 . However, it turns out that the three coefficients $C\left[\begin{array}{c}b_{1} \\ 1\end{array}\right], C\left[\begin{array}{c}b_{2} \\ 1\end{array}\right]$ and $C\left[\begin{array}{c}b_{3} \\ 1\end{array}\right]$ have no effects on the index of the $N=2$ supercoformal theory. So we are left with eight possibilities given by the values of the three coefficients $C\left[\begin{array}{l}b_{1} \\ b_{2}\end{array}\right]$, $C\left[\begin{array}{l}b_{1} \\ b_{3}\end{array}\right]$ and $C\left[\begin{array}{l}b_{2} \\ b_{3}\end{array}\right]$. These will give rise to eight models, but as far as the chiral asymmetry is concerned, there could be only two cases: (a) all these three coefficients are chosen to be the same; and (b) only two of these coefficients are the same, the third one differs by a sign. As an example of the first case, in our first model we choose

$$
C\left[\begin{array}{l}
b_{1} \\
b_{2}
\end{array}\right]=C\left[\begin{array}{l}
b_{1} \\
b_{3}
\end{array}\right]=C\left[\begin{array}{l}
b_{2} \\
b_{3}
\end{array}\right]=-1
$$

From (4.11) and (4.12), in this model we get $I_{b_{1}}=I_{b_{2}}=I_{b_{3}}=-16$, so the $N=2$ index is

$$
\operatorname{Tr}(-1)^{J_{0}^{\prime}}=I_{b_{1}}+I_{b_{2}}+I_{b_{3}}=-48
$$

This indicates that there are 48 generations in this model, a result that one could also derive by working out the massless spectrum of the model explicitly. In fact, in this model, each of the $b_{1}, b_{2}$ and $b_{3}$ produces two copies of space-time fermions, each copy transforming under the gauge group $S O(10) \times S O(6)^{3} \times E_{8}$ as follows

$$
\begin{aligned}
& b_{1}:(\mathbf{1 6}, \mathbf{4}, \mathbf{1}, \mathbf{1}, \mathbf{1})+(\mathbf{1 6}, \overline{\mathbf{4}}, \mathbf{1}, \mathbf{1}, \mathbf{1}) \\
& b_{2}:(\mathbf{1 6}, \mathbf{1}, \mathbf{4}, \mathbf{1}, \mathbf{1})+(\mathbf{1 6}, \mathbf{1}, \overline{\mathbf{4}}, \mathbf{1}, \mathbf{1}) \\
& b_{3}:(\mathbf{1 6}, \mathbf{1}, \mathbf{1}, \mathbf{4}, \mathbf{1})+(\mathbf{1 6}, \mathbf{1}, \mathbf{1}, \overline{\mathbf{4}}, \mathbf{1})
\end{aligned}
$$

Each of $b_{1}, b_{2}$ and $b_{3}$ provides us with $2(4+4)=16$ generations of chiral fermions which transform as $\mathbf{1 6}$ of $S O(10)$, and as a result we have a model with 48 families in total. It is interesting to note from the previous analysis that, $I_{b_{1}}, I_{b_{2}}$ and $I_{b_{3}}$, i.e., the contributions from the $b_{1}, b_{2}$ and $b_{3}$ sectors to the index $\operatorname{Tr}(-1)^{J_{0}^{\prime}}$, simply specify the number of chiral fermions contained in these sectors. As an example of the second case, we now consider our second model with

$$
C\left[\begin{array}{l}
b_{1} \\
b_{2}
\end{array}\right]=C\left[\begin{array}{l}
b_{1} \\
b_{3}
\end{array}\right]=-1, \quad C\left[\begin{array}{l}
b_{2} \\
b_{3}
\end{array}\right]=+1 .
$$

We get $I_{b_{1}}=-16$ and $I_{b_{2}}=I_{b_{3}}=0$ from (4.11) and (4.12), so we now have a model with only 16 generations instead. Again, this is precisely what one would derive by analyzing the massless spectrum of this model. In this model, the gauge group becomes $S O(10) \times$ $S O(6)^{3} \times S O(16)$, and none of the fermions that would arise from the $b_{2}$ and $b_{3}$ sectors survive the GSO-projections, so the $b_{1}$ sector is the only source of chiral fermions.

We now extend the basis $\mathcal{B}_{1}$ to $\mathcal{B}_{2}=\left\{\mathbf{1}, S, b_{1}, b_{2}, b_{3}, b_{4}, b_{5}\right\}$, by adding two more basis vectors $b_{4}$ and $b_{5}$ of the following form [16]

$$
\begin{aligned}
& b_{4}=\left(\begin{array}{llllllll}
1 & 100100010001001010: 001001000110 & 100 & 11111 & \mathbf{0}_{8}
\end{array}\right), \\
& b_{5}=\left(\begin{array}{llllll}
1 & 001010100100001010: 010001 & 10001001011111 & \mathbf{0}_{8}
\end{array}\right)
\end{aligned}
$$


Since $N_{b_{4}}=N_{b_{5}}=2$, the number of vectors in $\Xi^{-}$for the models which are generated by $\mathcal{B}_{2}$ increases to $M / 2=2^{7} / 2=64$.

In addition to the 16 sectors in (4.2), the 48 new sectors are:

$$
\begin{aligned}
& b_{4}(+\zeta), \quad b_{5}(+\zeta), \quad \mathbf{1}+b_{4}+b_{5}(+\zeta), \quad S+b_{4}+b_{5}(+\zeta), \\
& S+b_{1}+b_{2}+b_{3}+b_{4}(+\zeta), \quad S+b_{1}+b_{2}+b_{3}+b_{5}(+\zeta), \\
& b_{1}+b_{4}+b_{5}(+\zeta), \quad b_{2}+b_{4}+b_{5}(+\zeta), \quad b_{3}+b_{4}+b_{5}(+\zeta), \\
& S+b_{1}+b_{2}+b_{4}+b_{5}(+\zeta), S+b_{2}+b_{3}+b_{4}+b_{5}(+\zeta), S+b_{3}+b_{1}+b_{4}+b_{5}(+\zeta), \\
& b_{1}+b_{2}+b_{4}\left(b_{5}\right)(+\zeta), \quad b_{2}+b_{3}+b_{4}\left(b_{5}\right)(+\zeta), \quad b_{3}+b_{1}+b_{4}\left(b_{5}\right)(+\zeta), \\
& S+b_{1}+b_{4}\left(b_{5}\right)(+\zeta), \quad S+b_{2}+b_{4}\left(b_{5}\right)(+\zeta), \quad S+b_{3}+b_{4}\left(b_{5}\right)(+\zeta) .
\end{aligned}
$$

Among these 48 new sectors, only three sectors $\left(b_{4}, b_{5}, S+b_{4}+b_{5}\right)$ provide massless spacetime fermions. It is straightforward to check that Eq. (4.4) holds for any $\beta$ taken from the set (4.18), so $S$ and $S+\zeta$ still do not contribute to the index (3.8). Similarly, the contributions of $\left(b_{1}, b_{2}, b_{3}\right)$, all new terms for $\beta$ from set (4.18) vanish. However, because $M / 2$ increases by a factor of $4,(4.11)$ and (4.12) now become

$$
I_{b_{1}}=2\left(C\left[\begin{array}{l}
b_{1} \\
b_{2}
\end{array}\right]+C\left[\begin{array}{l}
b_{1} \\
b_{3}
\end{array}\right]\right), \quad I_{b_{2}}=8\left(C\left[\begin{array}{l}
b_{2} \\
b_{3}
\end{array}\right]+C\left[\begin{array}{l}
b_{2} \\
b_{1}
\end{array}\right]\right), \quad I_{b_{3}}=8\left(C\left[\begin{array}{l}
b_{3} \\
b_{1}
\end{array}\right]+C\left[\begin{array}{l}
b_{3} \\
b_{2}
\end{array}\right]\right) .
$$

Carrying out the same calculations for sectors $\left(b_{4}, b_{5}, S+b_{4}+b_{5}\right)$, we have

$$
I_{b_{4}}=I_{b_{5}}=I_{S+b_{4}+b_{5}}=0
$$

Therefore, given the basis $\mathcal{B}_{2}$, the choice (4.13) gives rise to 12 generations, while the choice (4.16) gives rise to 4 generations.

We would like to point out that the result (4.19) only depends on the form of the basis vectors $b_{4}$ and $b_{5}$ and does not depend on the choice of the spin-structure coefficients. As a consequence no matter how one chooses the relevant coefficients $C\left[\begin{array}{l}b_{i} \\ b_{j}\end{array}\right]$, in models generated by the basis $\mathcal{B}_{2}$, space-time fermions arising from the sectors $\left(b_{4}, b_{5}, S+b_{4}+b_{5}\right)$ are always vector-like. In fact, by analyzing the massless spectrum, one can find that, the fermions from sectors $b_{4}$ or $b_{5}$ always transform as $\mathbf{1 6}+\overline{\mathbf{1 6}}$ of $S O(10)$, while those from sector $S+b_{4}+b_{5}$ either transform as $\mathbf{1 0}$ or as singlets of $S O(10)$.

All the models we have considered above can be viewed upon bosonization as symmetric $Z_{2} \times Z_{2}$ orbifold models, for a discussion see e.g. Ref. [19]. As we have mentioned in the beginning of this section, these models in fact admit a $(2,2)$ world-sheet supersymmetry. To show that our index formula (3.8) in fact works for some $(2,0)$ models as well, we now would like to briefly discuss one such example, the detailed analysis of this and 
other models will be presented elsewhere [20]. In addition to the basis vectors in $\mathcal{B}_{2}$, we add one extra basis vector of the form [21].

$$
\alpha=\left(0000000000000000011: 000001011001 \frac{1}{2} \frac{1}{2} \frac{1}{2} \frac{1}{2} \frac{1}{2} \frac{1}{2} \frac{1}{2} \frac{1}{2} \frac{1}{2} \frac{1}{2} \frac{1}{2} \frac{1}{2} 1100\right) .
$$

This new basis vector breaks down the right-moving $(0,2)$ structure, and has order $N_{\alpha}=4$. With the basis $\mathcal{B}_{3}=\left\{\mathbf{1}, S, b_{1}, b_{2}, b_{3}, b_{4}, b_{5}, \alpha\right\}$, the set $\Xi^{-}$contains $M / 2=256$ vectors, namely, the 64 vectors of (4.2) and (4.18) and those which can be obtained by adding to them $\alpha, 2 \alpha$ and $3 \alpha$. Since this basis contains a $(2,2)$ "core", we can still use the 12 real fermions $\bar{y}^{I}, \bar{\omega}^{I}$ and the three complex fermions $\bar{\eta}^{1,2,3}$ as the right-moving internal degrees of freedom in our expression for the index (3.8), and carry out the calculation essentially the same way. However, because of the absence of the $(0,2)$ structure in the current case, the analysis turns out to be somewhat involved. For one thing, the contribution of each sector to the $N=2$ index, namely, each term in the $\alpha$-summation of (3.8) by itself is no-longer bound to be an integer. In spite of this fact, the cancellations which take place in the $\alpha$-summation always render the index to be an integer. Futhermore, the role of the spin-structure coefficients $C\left[\begin{array}{l}b_{i} \\ b_{j}\end{array}\right]$ becomes more important. For the choice given in Ref. [21], we get [20]

$$
\operatorname{Tr}(-1)^{J_{0}^{\prime}}=-3
$$

This is in complete agreement with the result that this model has three generations [21].

\section{Conclusions.}

In this last section we will summarize the results presented in this paper. The free fermionic string models represent a class of classical string vacua. Although they lack a geometric interpretation, they are particularly simple to build and as a result several realistic models have been constructed.

In string theory there seems to be an intimate connection between spacetime and world-sheet physics. In the spirit of this philosophy and in the context of free fermionic string models, we have associated the number of generations of a string vacuum with $N=1$ spacetime supersymmetry with the index of the supersymmetry generator of the underlying $N=2$ internal superconformal theory. The existence of $N=1$ spacetime supersymmetry appears to be vital in our attempts to define the index in terms of the zero mode of the $U(1)$ Kac-Moody current of the $N=2$ algebra and to interpret it as the number of generations of a string vacuum. The index depends on the internal fermions and the formula we derived in section 3 is sensitive only to the boundary conditions of the internal fermions and the coefficients which represent the weights with which each sector contributes to the one-loop partition function. We have also applied this formula to a number of realistic free fermionic models and have calculated the number of generations $N_{g}$ of these models. Furthermore we have verified our results by explicitly deriving the massless spectrum of these vacua. The index being a topological quantity is invariant 
under deformations of the superconformal structure. Thus, it characterizes a family of string vacua rather than a particular vacuum of string theory.

Finally one last observation is in order. The different sectors of the models contribute to the formula for the index powers of 2 . The origin of this phenomenon resides in the equivalence of free fermionic models with the $Z_{2} \times Z_{2}$ orbifold compactifications. The number of generations in orbifold models is related to the Euler character of the orbifold which equals the number of its fixed points (powers of 2 for $Z_{2}$ orbifolds). A generic orbifold model typically produces a large number of generations. In order to reduce the number one incorporates either the action of a discrete group with no fixed points or introduces Wilson lines. On the contrary in free fermionic models the number of generations is sensitive only to the boundary conditions of the fermions and to certain coefficients. By introducing extra basis vectors compatible with the necessary rules we can reduce the number of generations. As a result it is easier to incorporate their effect into a general formula like the one we have presented in section 3.

\section{Acknowledgments.}

We would like to thank I. Antoniadis and M. Evans for useful discussions. This work has been supported in part by DOE grant DE-FG05-91-ER-40633.

\section{References.}

[1] P. Candelas, G. Horowitz, A. Strominger and E. Witten Nucl. Phys. B258 (1985) 46.

[2] L. Dixon, J. Harvey, C. Vafa and E. Witten Nucl. Phys. B261 (1985) 678.

[3] C. Vafa Mod. Phys. Lett. A12 (1989) 1169; K. Intriligator and C. Vafa Nucl. Phys. B339 (1990) 95.

[4] A. Faraggi and D. V. Nanopoulos Phys. Rev. D48 (1993) 3288.

[5] C. Hull and E. Witten Phys. Lett. 160B (1985), 398; W. Boucher, D. Friedan and A. Kent Phys. Lett. 172B (1986), 316; A. Sen Nucl. Phys. B278 (1986), 289; T. Banks, L. Dixon, D. Friedan and E. Martinec Nucl. Phys. B299 (1988), 613.

[6] E. Witten Nucl. Phys. B202 (1982), 253.

[7] W. Lerche and N. Warner Phys. Lett. 205B (1988), 471.

[8] A. Schwimmer and N. Seiberg, Phys. Lett. 184B (1987) 191.

[9] H. Kawai, D.C. Lewellen and H. Tye, Phys. Rev. Lett. 57 (1986) 1832; Nucl. Phys. B288 (1987) 1.

[10] I. Antoniadis, C. Bachas and C. Kounnas, Nucl. Phys. B289 (1987) 87; I. Antoniadis and C. Bachas, Nucl. Phys. B298 (1988) 586.

[11] R. Bluhm, L. Dolan and P. Goddard, Nucl. Phys. B309 (1988) 330.

[12] I. Antoniadis, C. Bachas, C. Kounnas and P. Windey Phys. Lett. 171B (1986), 51.

[13] H. Dreiner, J.L. Lopez, D.V. Nanopoulos and D. Reiss, Nucl. Phys. B320 (1989) 401. 
[14] K. Yuan, Ph.D. Thesis, Texas A\&M University (1991).

[15] S. Kalara, J.L. Lopez and D.V. Nanopoulos, Nucl. Phys. B353 (1991) 650.

[16] I. Antoniadis, J. Ellis, J. Hagelin and D.V. Nanopoulos, Phys. Lett. 205B (1988) 459.

[17] S. Cecotti and L. Girardello, Phys. Lett. 110B (1982) 253.

[18] K. Narain, M. Sarmadi and C. Vafa, Nucl. Phys. B288 (1987) 551.

[19] J. Lopez, D.V. Nanopoulos and K. Yuan, Phys. Rev. D50 (1994) 4060.

[20] I. Giannakis, D. V. Nanopoulos and K. Yuan, CTP-TAMU preprint under preparation

[21] J. Lopez, D.V. Nanopoulos and K. Yuan, Nucl. Phys. B399 (1993) 645. 